CYBERNETICS AND INFORMATION TECHNOLOGIES • Volume 15, No 7

Special Issue on Information Fusion

Sofia $\bullet 2015$

\title{
Human Activity Registration Using Multisensor Data Fusion
}

\author{
Kiril Alexiev, Georgi Shishkov, Nevena Popova \\ Institute of Information and Communication Technologies, Bulgarian Acad. of Sci., Sofia, Bulgaria \\ Emails: \{alexiev, gshishkov,npopova\}@bas.bg
}

Abstract: The paper discusses the feasibility of using smart phone devices for human activity registration and analysis. The functional characteristics of the smart phones and their permanent connectivity allow them to serve as a measurement lab and processing unit. An example of using the smart phones as a sensor data source is described, and the corresponding algorithm and results are given. The possible problems are listed and commented.

Keywords: Smart phone, human activity registration.

\section{Introduction}

Nowadays the smart phones are conquering the world. Almost every one of the citizens on our planet has one or more intelligent personal communication devices. More than 1 billion smart phones were sold in 2013. Global smart phone sales have exceeded 1.2 billion in units in 2014, according to GfK [1]. eMarketer predicts that the mobile communication penetration will reach two thirds of the population before the end of 2015 [2]. The users of smart phones at that time will be more than 2 billion.

The answer to the question "Why has this happened?" is hidden in the multifunctionality of the smart phones. Of course, the smart phone is a mobile phone first of all, (a cell phone), but it acquires quick functionality of all other mobile devices and becomes an indispensible device for all of us. Its advanced mobile operating system allows it to possess many features of a personal computer with the whole diversity of the personal computer applications - to be a Personal Digital Assistant (PDA), media player, GPS navigation unit, digital camera device, mobile payment device, to use many net protocols like WiFi, Bluetooth, NFC (Near Field Communication) and others for connectivity with other people and devices. A big variety of sensors is imbedded in a smart phone. The usual set of sensors includes 
two digital cameras, a proximity sensor, a full 3D kit of inertial sensors (three accelerometers, three gyroscopes, three compass sensors), a pressure sensor, a temperature sensor, a microphone, a humidity sensor, etc. The user interface is enlightened by a touch screen with widened size (4"-6" in diagonal) and HD resolution.

The functional characteristics of the smart phones are defined most of all with respect to the computing power. The processors of Qualcomm dominate in the field of mobile devices and its latest SnapDragon processors are 64 bit with clock speed of $2.3 \mathrm{GHz}$. Their multicore architectures consist of up to 8 cores. Additionally, a graphics chip is used. In the case of a Nvidia Tegra graphic chip, there are 256 GPU cores. Usually the power consumption is limited up to $10 \mathrm{~W}$, but all of them have more power efficient rate with a little companion core. The computing power without enough memory could not be efficiently used. Smart phones use two types of memory - internal one and memory cards. The internal memory usually counts several GB, but in the most expensive models, it is $128 \mathrm{~GB}$. The memory cards extend the mobile device memory and additionally serve as a device for sharing and preserving susceptible information. Usually the size of the memory cards is from 2 GB up to 64 GB. The battery assures normal functioning of the mobile device for a certain period of time. In spite of the constantly increasing capacity of the batteries (today it is up to 4-5 A.h), there is not any device with busy time exceeding $24 \mathrm{~h}$.

The contemporary functional characteristics of smart phones allow them to serve as a real measurement device (lab) which is combined with powerful computational resources and suitable interface for communication with a person and remote servers! So, smart phones can measure and process gathered information even in real time and interact with the owner or with a remote place in case of an emergency situation. Due to the fact, that today the smart phone is a firm (irrevocable) attribute of modern man (more than 2 billion users), it can be used as a convenient device to study the lifestyle of people through permanent recording of the data from the respective sensors and real time data analysis or postponed analysis at non-busy time. For the first time in mankind history there is a really widespread device that can be used for objective feedback of the style of living. This opens up new opportunities for the assessment of some actions, behaviour estimation, behaviour consultancy, for detecting changes in health and many others. Moreover, the information gathered from the observed people may be summarized and exploited in order to produce estimates on more complex social issues and to produce the appropriate action aiming at enhancement of the quality of life. As a first step in this field, we will consider one relatively simple task - human activity registration and estimation. To solve the task, inertial sensing elements are mainly used and phone computational power as well. In the next section, the state of the art will be described. Then a short description of the used sensing elements will be given. The proposed robust algorithm for activity estimation will be described in the fourth section. The results of implementation of this algorithm on real measured data are depicted in many figures. The last section before the conclusion discusses the main obstacles in the usage of smart phones as activity registration devices. The conclusion summarizes the received results and gives some ideas for further research. 


\section{State of the art}

The human activity registration became popular in the recent decades, when many specialists in image processing and recognition were involved in this field of research [4]. Now there is a tremendous amount of papers, treating human activity recognition using most of all video sensors. Almost all of these papers proposed a method for detecting a different type of the human activity or for classification between several of them. The task is non-trivial because of the complexity and diversity of the human activities. Conceptually, it may be categorized as self-dependent activity, humanobject activity, human-human activity, group activities, etc. Even if we confine to the individual activity of a man, there are various types, like gestures, different actions (sleeping, sitting, standing, walking, running, riding, climbing, etc.). Obviously, the variety of all possible types of human activities is vast enough to be covered by only one sensor set.

The theory of inertial systems and inertial navigation has appeared at the end of 18th century with the fundamental Euler book "The Theory of Motion of Solids", where for the first time, the spinning top effect was discussed. In 1852 the French scientist and physicist Leon Foucault has invented the gyroscope. Germany put into practice the first gyro-compass in 1909. During the Second World War the dreadful German V2 rockets were equipped with two gyros and one accelerometer. In 1958 an entirely standalone inertial navigation system was mounted on a submarine and it gave a position error of 10 miles for 97 hours (1830 miles distance). The technological advances in 1970-ies allowed mass production of miniature inertial sensors with a reduced form factor and smaller power consumption. The advantages of the inertial sensing elements, compared to other sensors were shown in a review of the human motion registration [5]. At this time the accelerometers and gyros in MEMS (Micro-Electro Mechanical System) realization were applied in a wearable system for human activity monitoring.

The first trial to use a cell phone for activity registration dates since 2007 year, when Nokia N95 phone has appeared. This phone can be regarded as one of the first smart phones. It was equipped with an accelerometer sensor, used for video stabilization and automatic photo orientation. Later Nokia has released a step counter application and has allowed access to the accelerometer from application software. In [6] the authors have used the accelerometers, embedded in smart phones, for classification of six activities, like walking, jogging, climbing stairs, sitting and standing. Five other activities (walking, running, sitting, standing, and lying) are classified, using unsupervised learning by the authors in [7]. A detailed picture of the smart phone implementation for human activity registration is given in [3].

Two attractive features of the smart phone have determined our choice in favour of its use as a measuring device:

- The smart phone is unobtrusive! People take them eagerly, at every opportunity, everywhere. It makes them ubiquitous $\square \square$ this assures continuous service. It does not violate human normal activities. Even in the case when the sensors are wearable, some people may feel a sense of inconvenience due to the contact to the body. 
- The comunication capabilities of a smart phone are excellent. The smart phone may be easily connected to almost all networks; it supports several types of protocol suits. The human interface is also optimally designed due to multimillion practice.

\section{The smart phone as a measurement lab}

Contemporary smart phones are equipped with a complete set of sensors for inertial navigation. The sensor set includes gyro sensors, accelerometers and magnetometers. The gyro sensor has a little misleading name. It remained from the beggining of the inertial navigation, when real gyroscopes were used. The gyroscopes keep the same attitude in space and the current orientation of the body is measured with respect to their permanent orientation. Now, all sensors in smart phones are MEMS and they are strapped to the phone body. Gyro sensors measure the rotation rate of the body. Through integration, the angles of rotation are received and the current phone attitude is calculated with respect to the initial one. The accelerometer provides information about the linear acceleration of the body. Their measurements always contain gravity acceleration. To extract the pure acceleration of the phone body, gravity acceleration has to be removed. Double integration of the accelerometers data allows us to receive the position of the phone body with respect to some initial position. Both types of sensors, above described, are used to calculate the body attitude and position based on some knowledge about the initial/previous attitude and position. This approach is called dead reckoning in navigation. The main advantage of the approach is that it will not need any other information, except sensor data. But the dead reckoning method has an obnoxious drawback to accumulate the errors.

The set of sensors for inertial navigation usually contains magnetometers. These sensors measure the strength of the magnetic field and they serve as a digital compass that gives the current phone orientation with respect to the Earth magnetic field. In spite of their inaccuracy, due to disturbances in the magnetic field, these sensors do not accumulate errors.

The inertial sensor set is complemented by a GPS receiver. If the satellite signals are accessible, GPS sensor gives the absolute position of the phone on the Earth.

Usually, 3D motion description of the body is given by three orthogonally placed accelerometers, giving the transition dynamics of the body and three orthogonally placed gyro sensors, determining the orientation/attitude of the body. The axes of both types of the sensors normally coincide, e.g., in a 3D orthogonal coordinate system there are sensors to measure the linear accelerations on each of the axes and the rotation rate on the same axes.

In the period of algorithm development and testing, transmission of the sensor data from the smart phone and computer must be organized. There are several ways to receive sensor data on a remote computer. The simplest one is to save the sensor data in the phone memory and after the experiment the recorded data stream is transferred through USB cable. The second one assures data transmission in real time. The transmission protocol is WiFi. A laptop is configured as a server and the phone is a client. To increase the transmission rate, UDP protocol is used. It is accepted that 
the channel is robust enough and there is no need to be confirmed at receiving every packet of data. The third method for data transmission considers again data recording on the phone. The application Dropbox is activated. Dropbox automatically synchronizes the files between your desktop computer and the phone. When the data files are saved in the Dropbox folder on your mobile device, it immediately downloads information to the Dropbox directory on the desktop computer. One of the available freeware applications for access to the sensor set on Android is "Sensorstream IMU+GPS". In Fig. 1 the option panel of this program for parameter setting of the sensor data stream is given. On the second panel (Fig. 2), the appropriate set of sensors may be chosen.

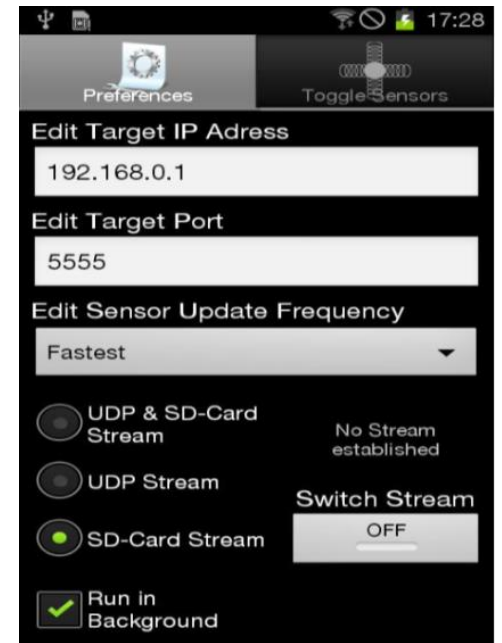

Fig. 1. Option panel of the sensor stream application

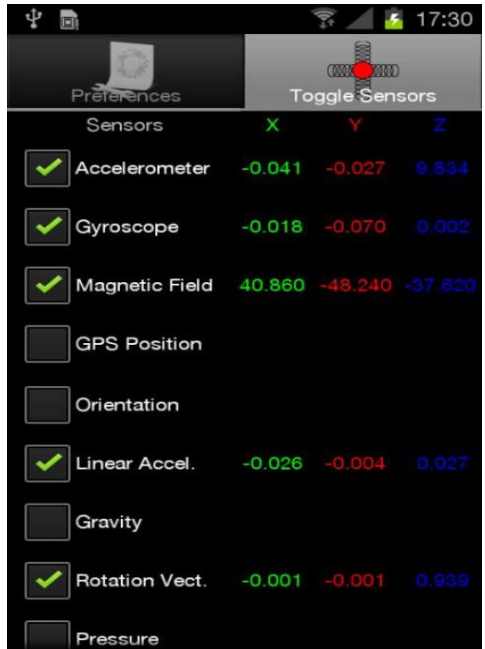

Fig. 2. Sensor panel of the sensor stream application

Most of the embedded sensors in smart phones present low cost hardware and their accuracy characteristics are far from the best ones. Summarizing the information of this section, it is clear that the sensor set in the mobile phones may serve as a source of information of the human activity, but after careful analysis of the tasks to be solved. This is especially valid for inertial sensors, where due to the inaccuracy of the sensing elements and initial misalignment, the errors are accumulated and soon after the algorithm starts, the results become compromised. To avoid such a situation, a more accurate sensor is required or design of the so called aided multisensor system, where several sensors complement each other. The joint use of various sensors for solution of different tasks is called sensor data fusion.

\section{Algorithm description}

One of the most challenging problems concerns energy expenditure as a measure of the physical activity. The so called doubly-labeled water technique is a well established method, regarded today as a reference for any other instruments for energy expenditure. In spite of its noninvasive procedure, it is too complicated due 
to the necessity for stable isotopes of hydrogen and oxygen and mass spectrometry for isotope concentration measurement. The isotopes production and analysis are expensive and require laboratory setup and special skills. The method above given can be applied for most human activities.

In our experiment we simplify radically the problem and will regard only the walking activity. An estimate of the energy expenditure will be received through calculation of the completed work for lifting a weight equal to the weight of the person at each step. In Figs 3-6 the signals, received by different sensors are indicated - accelerometers, gyros, magnetometers and spectrum of the accelerometer. The signals received are for a man, walking back and forth.

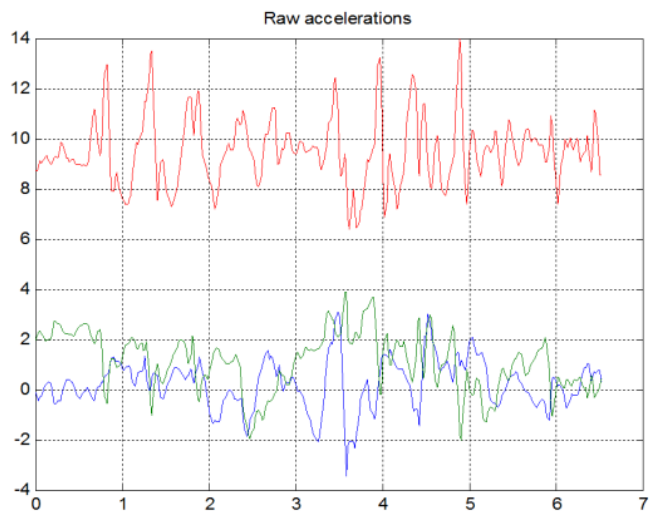

Fig. 3. Accelerometers data

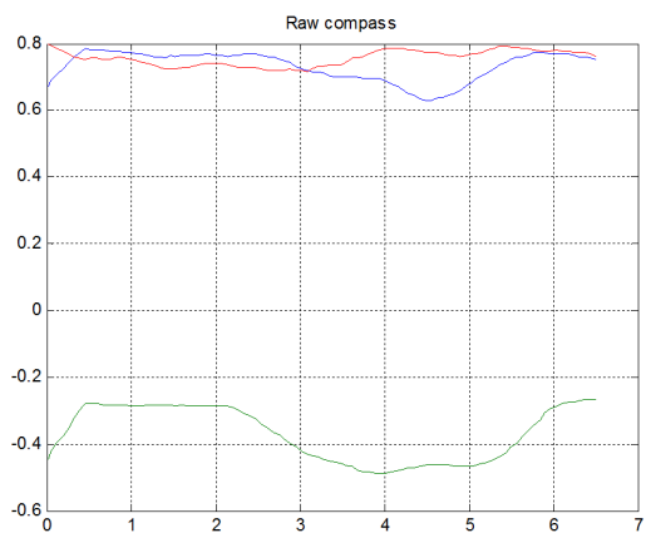

Fig. 5. Compass measurements

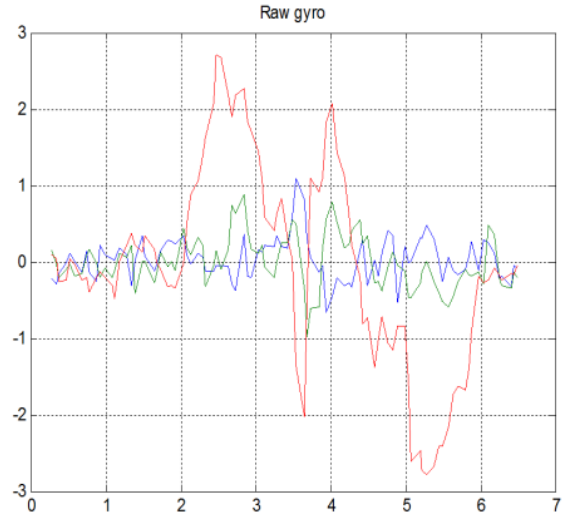

Fig. 4. Gyro data

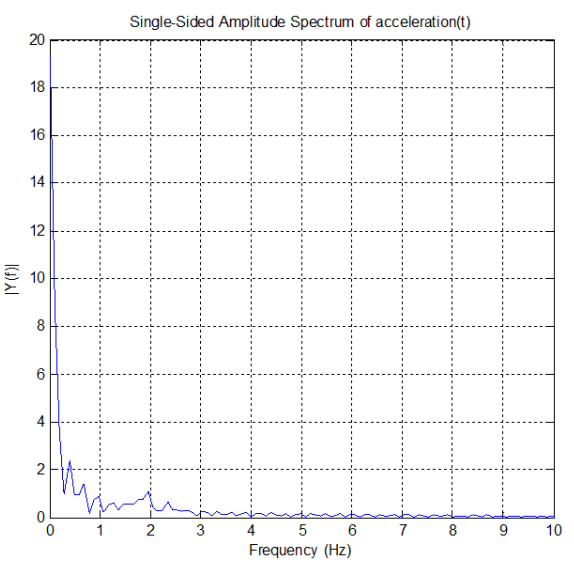

Fig. 6. Acceleration spectrum

The accelerometer data clearly show regular oscillations, because of walking. The gyro data has subtle oscillations and a big one, when there is a change of the direction. The magnetometer data (Fig. 5) definitely cannot be useful in our task. The spectrum of the accelerometer signal shows that most of the energy is concentrated below $3 \mathrm{~Hz}$. It is natural the spectrum energy of walking activity to be at low frequencies.

Let us now express the work, done for lifting the weight of a person: 


$$
W=\int F(t) \cdot s \cdot \cos (\varphi(t)) d t .
$$

It is considered that the work is linearly proportional to the energy expenditure. Due to the change of magnitude and direction of the applied force $F$, we have to use an integral. In this equation $s$ denotes the height, at which the weight is lifted, $\varphi$ is the angle between the applied and vertical force, $t$ is time. Applying Newton's second low of motion we can rewrite the above equation like:

$$
W=\int m a_{p}(t) \cdot s \cdot \cos (\varphi(t)) d t,
$$

where $m$ is the mass of the person, $a_{p}$ is the resulting acceleration of the applied force. We reorganize the equation in a more convenient form:

$$
\begin{gathered}
W=\frac{m g}{|g|} s \int\left(\frac{\vec{a}(t) \cdot \vec{g}}{|g|}-|g|\right) d t=\frac{\text { Weight }}{|g|} s \int\left(\frac{\vec{a}(t) \cdot \vec{g}}{|g|}-|g|\right) d t= \\
=k \cdot \text { Weight } \int\left(\frac{\vec{a}(t) \cdot \vec{g}}{|g|}-|g|\right) d t .
\end{gathered}
$$

The last equation gives with accuracy up to a coefficient the quantity of work, or the linear relationship with energy expenditure. The expression in the integral is a vector product of the total acceleration vector and gravity vector.

It is considered that the smart phone can be kept in the pocket of the user's pants, in a bag, on the waist or even in the hand. In the general case the smart phone will be a subject of additional impacts. We accept the assumption that all impacts with frequency higher than $3 \mathrm{~Hz}$ are not caused by the person. In order to remove them, a low pass Butterworth filter is applied. This filter has maximally flat frequency response in the pass band and the signal disturbancies as a negative consequence of the filtering procedure will be minimal. The cutoff frequency is $3 \mathrm{~Hz}$ and the chosen order of the filter is 10 . The total raw acceleration and the filtered acceleration are given in Figs 7 and 8 correspondingly.

To calculate the integral value, the direction of the gravity vector has to be found. This is not a new problem and all AHRS (Attitude and Heading Reference Systems), implemented in aviation, do this. Usually, the algorithms for AHRS use all nine sensors (accelerometers, gyros, magnetometers) and fuse their measurement. To simplify the algorithm, we assume that the direction of the gravity vector is given when the total acceleration is very close to $9.81 \mathrm{~m} / \mathrm{s}^{2}$. The algorithm makes a check of the closeness of the magnitude of total acceleration to $g$ and if this condition is fulfilled, the direction of $g$ is regarded the same as the direction of the total acceleration at this moment. This direction of $g$ is used until the next one is found. The scalar product of the total acceleration and gravity vector gives the magnitude of the vertical acceleration. To receive pure acceleration without gravity acceleration, a gravity magnitude has to be subtracted. The pure vertical acceleration is shown in Fig. 9.

The last step in data processing locates the extremes of the function and the part for integration (Figs 10 and 11). 


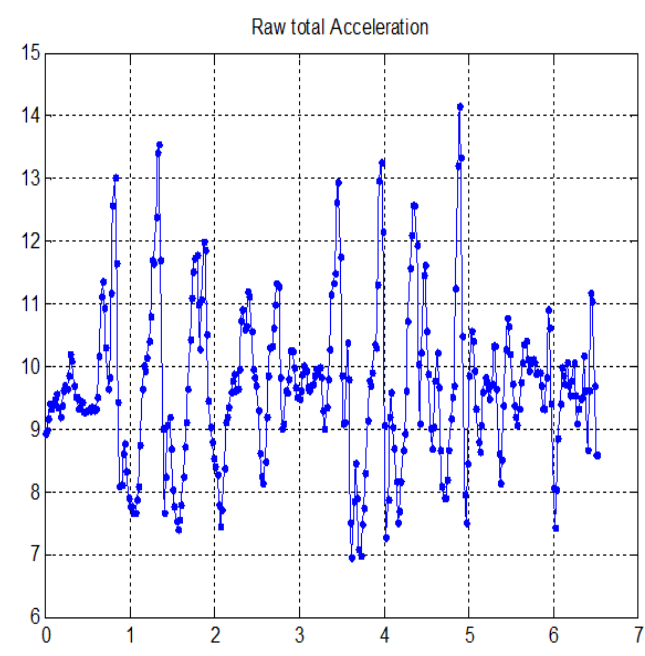

Fig. 7. Raw total acceleration

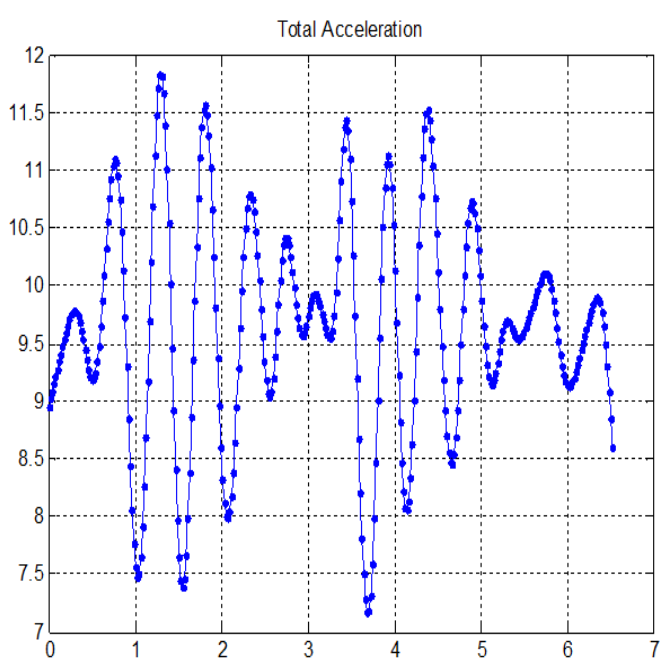

Fig. 8. Filtered total acceleration

The calculation of the work/energy expenditure opens the door to different kinds of analysis. Here only few of them will be mentioned. The acceleration corresponds to the force applied. The maximum/minimum of the acceleration characterizes the potential physical capabilities of a man. The smootness of walking can also be characterized through timing variability of the accelerometer signal (time-frequency analysis). The steepness of the change gives another physical characteristic. When GPS signal is available, all these measures can be normalized over the distance. The accelerometer data can serve as an estimate of the current human condition; they can show the tendency of future fatal events, like risk of falling, etc. The results can be aggregated on daily, weekly, monthly and even yearly bases for a person, for a group of persons, for society.

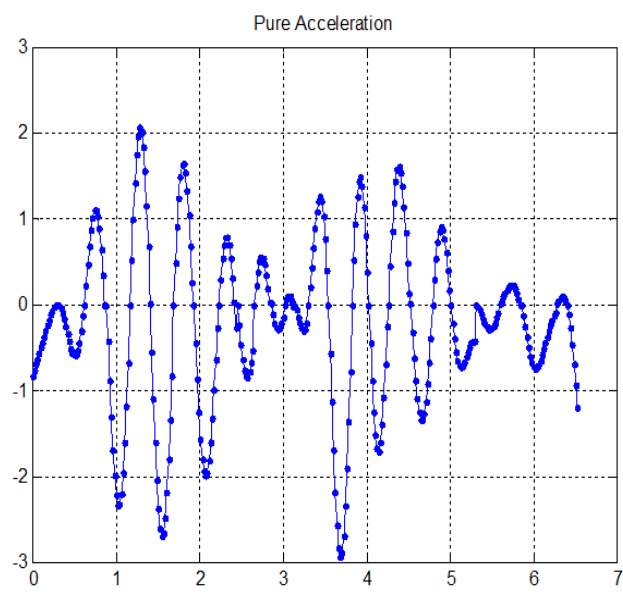

Fig. 9. Pure activity acceleration

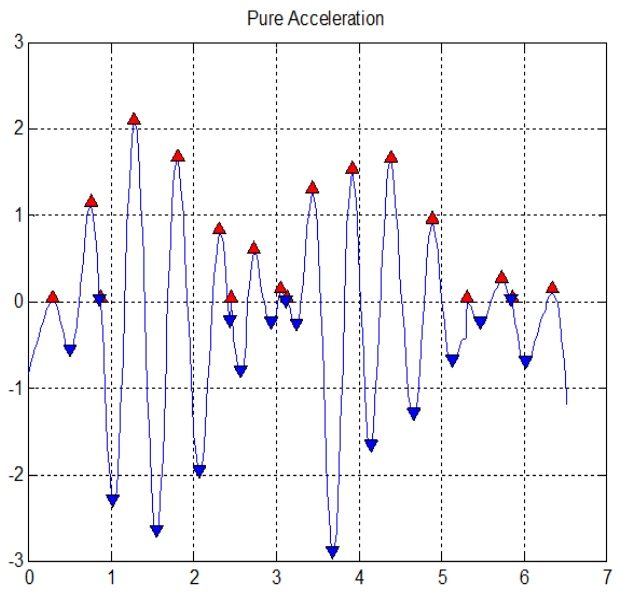

Fig. 10. Detected extrema 


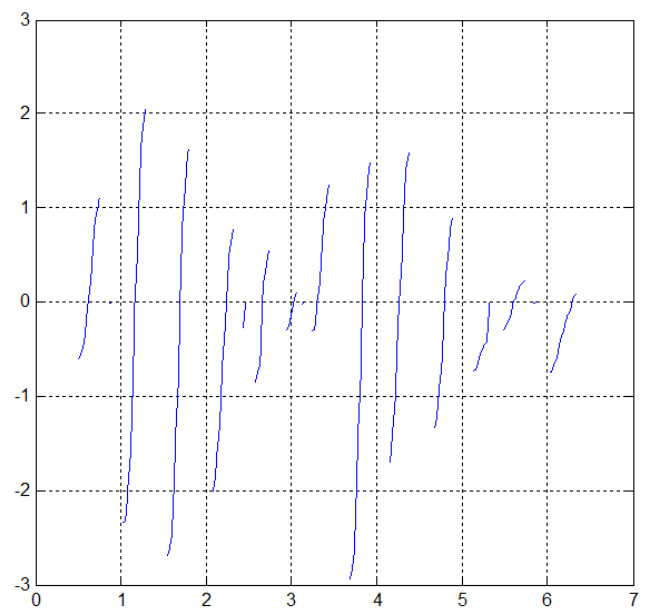

Fig. 11. Parts for integration

\section{The most important problems in the implementation of smart phones as measuring devices}

The latest smart phones have powerful processors, fast memory, plenty of sensors. They can easily serve as a real sensor data source to support human activity registration. The main drawback of the contemporary systems is the low quality of available hardware. To reduce the price of the handheld devices, the developers often go to solutions with low accuracy sensors. But the progress in the field is very big and soon the enhanced sensor sets will find place in the mobile devices.

There are several other issues that need a comment. One of them is where the smart phone is found. Different smart phone positions give different results, sometimes big disturbances are generated due to the free phone movement in the bag, for example. The battery limitation is also a substantial problem. In the power saving mode the phone remains active for weeks, but in a regime of permanent monitoring with one or more sensor sets and communication, the busy time is shortened essentially.

\section{Conclusion}

The research presented in this paper gives a proposal for using smart phones as human activity registration devices. A simplified algorithm for energy expenditure estimation is suggested as an example of real time application. It is shown that the sensor data contains information about the human physical activity, behavioural pattern for a prolonged period of time and some important parameters about the current health status of an individual and its change in time. The authors are interested in the usefulness of the smart phones as a platform for mass research of the human activity. 
Acknowledgements: This research is supported by AComIn Project "Advanced Computing for Innovation" of IICT-BAS (2012-2016), Grant 316087, funded by FP7 Capacity Program, (www.iict.bas.bg/acomin/).

\section{References}

1. https://www.gfk.com/news-and-events/press-room/press-releases/pages/global-smartphonesales-exceed-1-2b-units-in-2014.aspx

2. http://www.emarketer.com/Article/Smartphone-Users-Worldwide-Will-Total-175-Billion2014/1010536

3. Orti z, J. L. R. Smartphone-Based Human Activity Recognition. Springer Theses, Springer International Publishing Switzerland, 2015. DOI 10.1007/978-3-319-14274-6_1.

4. A g g a rwal 1, J. K., M. S. R y o o. Human Activity Analysis: A Review. ACM Computing Surveys (to appear).

5. Y a n g, C.-C., Y.-L. H s u. A Review of Accelerometry-Based Wearable Motion Detectors for Physical Activity Monitoring. - Sensors, Vol. 10, 2010, pp. 7772-7788.

6. Kwa pis z, J. R., G. M. We is s, S. A. Moore. Activity Recognition Using Cell Phone Accelerometers. - SIGKDD Explorations Newsletter, Vol. 12, 2010, No 2, pp. 74-82.

7. $\mathrm{K} w \mathrm{o}$, Y., K. K a n g, C. B a e. Unsupervised Learning for Human Activity Recognition Using Smartphone Sensors. - Elsevier, Expert Systems with Applications, Vol. 41, 2014, pp. 6067-6074. 